\title{
Approximate Completed Trace Equivalence of Three Dimensional t-Model Nonlinear Algebraic Hybrid Systems
}

\author{
Hao Yang ${ }^{1}$, Jinzhao $\mathrm{Wu}^{2, *}$, Zhiwei Zhang ${ }^{1}$ and Yang Liu ${ }^{1}$ \\ ${ }^{1}$ Chengdu Institute of Computer Application, Chinese Academy of Sciences, China \\ ${ }^{2}$ Guangxi Key Laboratory of Hybrid Computation and IC Design Analysis, Guangxi University for Nationalities; School of Computer \\ and Information Technology, Beijing Jiaotong University, China
}

Received: 23 Dec. 2012, Revised: 26 Apr. 2013, Accepted: 29 Apr. 2013

Published online: 1 Sep. 2013

\begin{abstract}
In order to optimize programs of three dimensional t-model nonlinear algebraic Hybrid Systems and eliminate system states, approximate completed trace equivalence of three dimensional t-model nonlinear algebraic Hybrid Systems is proposed. Firstly, the three dimensional t-model nonlinear algebraic program is used to describe the system continuous transition behavior. Then, the approximate of three dimensional t-model nonlinear algebraic Hybrid System is established. Whether trace is approximate or not could be decided through three dimensional t-model nonlinear algebraic Hybrid System trace approximate algorithm. Next, it put forward the approximate completed trace equivalence of three dimensional t-model nonlinear algebraic Hybrid Systems. It can optimize three dimensional t-model nonlinear algebraic program as well as reduce the number of system states. The experiment result shows that this method is effective.
\end{abstract}

Keywords: Three dimensional t-model nonlinear algebraic program, three dimensional t-model nonlinear algebraicHybrid Systems, completed trace equivalence

\section{Introduction}

Hybrid systems $[1,2,3,4]$ have both the characteristics of continuous systems and discrete systems. They have the continuous variables dynamic processes and discrete event processes. For the experimental techniques and methods are not perfect, the three dimensional t-model nonlinear algebraic Hybrid Systems always have errors. For the given permission precision, approximate analyse between real three dimensional $\mathrm{t}$-model nonlinear algebraic Hybrid Systems and approximate three dimensional t-model nonlinear algebraic Hybrid Systems is very significant. In the traditional functional analysis domain, equivalence relation establishes a standard among different systems. Completed trace equivalence [5, 6] is a common equivalence relation. Completed trace equivalence can reduce the number of system states. For the three dimensional t-model nonlinear algebraic Hybrid Systems, it does not have the corresponding approximate completed trace equivalence theory. The approximate completed trace equivalence of three dimensional t-model nonlinear algebraic Hybrid Systems which is proposed in this paper can optimize three dimensional t-model nonlinear algebraic program as well as reduce the number of system states.

The rest of this paper is organized as follows: In section 2, three dimensional t-model nonlinear algebraic Hybrid Systems are proposed. We also analyze the transition of three dimensional t-model nonlinear algebraic Hybrid Systems. In section 3, the approximate of three dimensional t-model nonlinear algebraic Hybrid Systems is established. Through three dimensional t-model nonlinear algebraic Hybrid System trace approximate algorithm, we decide whether two three dimensional t-model nonlinear algebraic Hybrid Systems is approximate or not. In section 4, approximate completed trace equivalence of three dimensional t-model nonlinear algebraic Hybrid System is put forward. It can optimize three dimensional $\mathrm{t}$-model nonlinear algebraic program as well as reduce the number of system states. In

\footnotetext{
* Corresponding author e-mail: $415360889 @$ qq.com
} 
section 5, above view is verified by the table tennis motion system example. In section 6 , we conclude the paper.

\section{Three Dimensional t-Model Nonlinear Algebraic Hybrid Systems}

Definition 1. (Three Dimensional t-Model Nonlinear Algebraic Program) Let $\mathrm{R}$ be the set of real numbers, $x_{i} \in R(i=1,2,3)$ and $x_{i}^{\prime} \in R(i=1,2,3)$ be the variables, $t \in R$ be a time variable. A three dimensional t-model nonlinear algebraic program is a algebraic program likes $X^{\prime}=X+F(t)$, where $X=\left(x_{1}, x_{2}, x_{3}\right)$ and $X^{\prime}=\left(x_{1}^{\prime}, x_{2}^{\prime}, x_{3}^{\prime}\right)$ are the pre- and post- state values of the three dimensional t-model nonlinear algebraic program transition. Let $F(t)=\left(f_{1}(t), f_{2}(t), f_{3}(t)\right)^{T}$ be a three dimensional column vector, where $f_{i}(t)(i=1,2,3)$ is a monadic polynomial and its constant term is zero. is monotonous and at least includes a monadic polynomial whose number of times is bigger than two.

Definition 2. (Three Dimensional t-Model Nonlinear Algebraic Hybrid System) A three dimensional t-model nonlinear algebraic Hybrid Systems is a tuple $H=<Q, V, H X$, Init , Lab, E, Inv, $F, R>$, where

(1) $Q$ is a set of system discrete locations.

(2) $V=x_{1}, x_{2}, x_{3}$ is a set of system continuous variables.

(3) $H X$ is a set of system continuous variables values.

(4)Init $\subseteq Q \times H X$ is initial state set of system. $Y_{0}$ is a system initial state value set and a three dimensional convex polyhedron.

(5) $L a b$ is a set of discrete transition programs. A discrete transition program is a homogeneous linear algebraic program $X^{\prime}=A X$ or an inhomogeneous linear algebraic program $X^{\prime}=A X+b$.

(6) $E \subseteq Q \times L a b \times Q$ is a set of discrete transitions.

(7)Inv is a set of continuous variables invariant set.

(8) $F$ is a set of three dimensional t-model nonlinear algebraic programs which describes system continuous variables dynamic processes.

(9) $R$ is a set of discrete location transition conditions. The set of discrete location transition conditions is composed by surfaces of invariant sets.

Definition 3.(Three Dimensional t-Model Nonlinear Algebraic Hybrid System ?Transition) A state $s$ of three dimensional t-model nonlinear algebraic Hybrid System is a pair $\langle q, H X\rangle$, where $q \in Q$ is a discrete location. A state $\langle q, H X\rangle$ is said to be admissible if $X \in \operatorname{Inv}(q)$

Let $\rightarrow C$ be the continuous transition, in the time period $\left[t_{i-1}, t_{i}\right]$, if there exists a three t-model nonlinear algebraic program $X^{\prime}=X+F(t)$, $X_{1}=X\left(t_{i-1}\right)=X_{0}+F\left(t_{i-1}\right)$, $X_{2}=X\left(t_{i}\right)=X_{0}+F\left(t_{i}\right), \forall t \in\left[t_{i-1}, t_{i}\right], X(t) \in \operatorname{Inv}(q)$,then $\left(q, X_{1}\right) \rightarrow C\left(q, X_{2}\right)$, where $X_{0}$ is a initial state value of current discrete location.

Let $\rightarrow D$ be the discrete transition, if $e=<q_{1}, l a b, q_{2}>\in E$ and $\left.\left(X_{1}, X_{2}\right) \in R(e)\right)$ then $\left(q_{1}, X_{1}\right) \rightarrow D\left(q_{2}, X_{2}\right)$, where $R(e)$ is a set of discrete location transition conditions and $X_{2}=A X_{1}$ or $X_{2}=A X_{1}+b$.

At the time $t$, the reachability value state set which is from the initial state value set $Y_{0}$ is defined as

$$
R_{t}\left(Y_{0}\right)=\left\{X_{t} \mid X_{t}=X\left(t, X_{0}\right), \exists X_{0} Y_{0}\right\} .
$$

In the time period $\left[t_{0}, t_{f}\right]$, the reachability state value set which is from the initial state value set $Y_{0}$ is defined as

$$
R_{\left[t_{0}, t_{f}\right]}\left(Y_{0}\right)=\bigcup_{t \in\left[t_{0}, t_{f}\right]} R_{t}\left(Y_{0}\right)
$$

\section{Approximate of Three Dimensional t-Model Nonlinear Algebraic Hybrid Systems}

At present, there does not exist flow pipeline accurate calculation method. It has only flow pipeline approximate calculation method $[7,8]$. If the convex initial state value set $Y_{0}$ and time period $\left[t_{0}, t_{f}\right]$ are given, then it computes the polyhedral over approximation $\hat{R}_{\left[t_{0}, t_{f}\right]}\left(Y_{0}\right)$ of reachability state $R_{\left[t_{0}, t_{f}\right]}\left(Y_{0}\right) . \hat{R}_{\left[t_{0}, t_{f}\right]}\left(Y_{0}\right)$ can be expressed as a polyhedron $\bigcap_{i}\left\{X \mid c_{i}^{T} X \leq d_{i}\right\}$ which is enclosed of $m$ planoids.We can get $R_{\left[t_{0}, t_{f}\right]}\left(Y_{0}\right) \subseteq \hat{R}_{\left[t_{0}, t_{f}\right]}\left(Y_{0}\right)$, where $(C, d) \in R^{m \times n} \times R^{m}$, a row vector $c_{i}^{T}(i=1,2, m)$ of $C$ presents an outward unit normal vector of $\mathrm{i}$-th planoid, $d$ presents the distance between this planoid and origin.

In the time period $\left[t_{k-1}, t_{k}\right]$, the step of single stage flow pipeline over approximate algorithm is as follows:

(1)The initial state value set $Y_{0}$ is a convex polyhedron. It can get the $h$ vertexes of convex polyhedron. According to the three dimensional t-model nonlinear algebraic program, it obtains the corresponding vertexes at the time $t_{k-1}$ and time $t_{k}$ of $h$ initial vertexes. These corresponding vertexes connect into a convex polyhedron.

(2)An outward unit normal vector in the surfaces of convex polyhedron is a row vector of matrix $C$. This is the necessary condition for computing flow pipeline over approximation.

(3)Under the premise of matrix $C$ has been found, using optimization method with constraint conditions can calculate the convex polyhedron which has the smallest volume. $d_{k}^{\max }=\max _{X} c X$ satisfies the constranit condition $X \in R_{\left[t_{k-1}, t_{k}\right]}\left(Y_{0}\right) \quad$ can be written as $d_{k}^{\max }=\max _{X_{0}, t} c X\left(t_{0}, Y_{0}\right)$ satisfies the constranit condition $X_{0} \in Y_{0}$ and $t \in\left[t_{k-1}, t_{k}\right]$.

The $d_{k}^{\max }$ and matrix $C$ can be calculated by software MATLAB $[9,10]$.

In the time period $\left[t_{k-1}, t_{k}\right]$, the step of three dimensional over approximate flow pipeline $\hat{R}_{\left[t_{k-1}, t_{k}\right]}\left(Y_{0}\right)$ volume algorithm is as follows:

(1)Let $t_{0}=t_{k-1}, t_{f}=t_{k}$.

(2)In the time period $\left[t_{0}, t_{f}\right]$, if the $h$ vertexes $v_{1}(0), v_{2}(0), \ldots, v_{h}(0)$ of initial state value set $Y_{0}$ and the 
three dimensional t-model nonlinear algebraic program which are in the current invariant set is given, then it obtains $m$ corresponding vertexes $v_{1}\left(t_{0}\right), v_{2}\left(t_{0}\right), \ldots, v_{h}\left(t_{0}\right)$ at the time $t_{0}$ and $m$ corresponding vertexes $v_{1}\left(t_{f}\right), v_{2}\left(t_{f}\right), \ldots, v_{h}\left(t_{f}\right)$ at the time $t_{f}$.

(3)According to $\mathrm{m}$ corresponding vertexes $v_{1}\left(t_{0}\right), v_{2}\left(t_{0}\right), \ldots, v_{h}\left(t_{0}\right)$ at the time $t_{0}$ and $m$ corresponding vertexes $v_{1}\left(t_{f}\right), v_{2}\left(t_{f}\right), \ldots, v_{h}\left(t_{f}\right)$ at the time $t_{f}$., the initial three dimensional flow pipeline volume $V_{\alpha}$ in the time period $\left[t_{0}, t_{f}\right]$ is computed by convex polyhedron volume algorithm.

(4)The three dimensional over approximate flow pipeline is obtained with the flow pipeline over approximate algorithm, the three dimensional over approximate flow pipeline volume $V_{\beta}$ in the time period $\left[t_{0}, t_{f}\right]$ is computed by convex polyhedron volume algorithm.

(5)For a given permission precision $\sigma$, if $V_{\beta} / V_{\alpha}<1+$ $\sigma$, then the over approximate flow pipeline volume $V \beta$ is treated as three dimensional flow pipeline volume in the time period $\left[t_{0}, t_{f}\right]$. Otherwise it takes the center point $\frac{t_{0}+t_{f}}{2}$ of $\left[t_{0}, t_{f}\right]$. $\left[t_{0}, \frac{t_{0}+t_{f}}{2}\right]$ and $\left[\frac{t_{0}+t_{f}}{2}, t_{f}\right]$ are new time period.

(6)Each section over approximate flow pipeline volume in the time period $\left[t_{k-1}, t_{k}\right]$ adding together is recorded as $V L . V L$ is treated as over approximate flow pipeline $\hat{R}_{\left[t_{k-1}, t_{k}\right]}\left(Y_{0}\right)$ volume.

In the real life, the three dimensional t-model nonlinear algebraic program $X^{\prime}=X+F(t)$ is obtained after the measurement or calculation. $F(t)$ often has measurement errors or calculation errors. The real three dimensional t-model nonlinear algebraic program which we have to deal with is $X^{\prime}=X+[F(t)+\delta F(t)]$. Next, we will study the relation between real three dimensional t-model nonlinear algebraic program and approximate three dimensional $t$-model nonlinear algebraic program.We do not study the approximate of discrete transition programs. We start with initial state area $S_{0}$ of three dimensional t-model nonlinear algebraic Hybrid System to research approximate of three dimensional t-model nonlinear algebraic Hybrid System.

Let $Y_{0}$ be the initial state value set, in a transition route, the system passes through in turn discrete locations $q_{0}, q_{1}, \ldots, q_{k}$. At the discrete location $q_{j}(0 \leq j \leq k)$, the real three dimensional t-model nonlinear algebraic program is $X^{\prime}=X+[F(t)+\delta F(t)]$, the approximate three dimensional t-model nonlinear algebraic program is $\left.X^{\prime}=X+F_{j}(t)\right]$, the real invariant set and approximate invariant set is $I n v_{j}$. It is setting every passing time $T$ to calculate flow pipeline. At the qi, flow pipeline computation total time is $h_{j} T$. Let discrete transition program be $l a b_{i}(i=1,2, k)$, real initial state value set in $q_{i}$ be $Y_{i}^{\prime}$, approximate initial state value set at $q_{i}$ be $Y_{i}$. Let $g=1, \ldots, h_{j}$, in the time period $[(g-1) T, g T]$ at the $q_{j}$, the real continuous transition program $l_{j g}^{\prime}$ is

$$
X^{\prime}=X+\left[F_{j}(t)+\delta F_{j}(t)-F_{j}(g T)-\delta F_{j}(g T)\right]
$$

the approximate continuous transition program $l_{j g}$ is $X^{\prime}=X+\left[F_{j}(t)-F_{j}(g T)\right]$. We use three dimensional over approximate flow pipeline volume algorithm to judge real trace and approximate trace whether approximate or not. Before the system leaves the $q_{j}$, all discrete locations approxiamte flow pipeline volume sum of real system is written as $V_{m}^{\prime}$, all discrete locations approxiamte flow pipeline volume sum of approximate system is written as $V_{m}$. At the $q_{j}$, the over approximate flow pipeline volume of real system is written as $V L_{j}^{\prime}$, the over approximate flow pipeline volume of approximate system is written as $V L_{j}$.

The step of three dimensional $\mathrm{t}$-model nonlinear algebraic Hybrid System trace approximate algorithm is as follows:

(1)Let $V_{0}^{\prime}=0, V_{0}=0, n=0$.

(2)It computes the initial state value set $Y_{n}^{\prime}$ of real system and the initial state value set $Y_{n}$ of approximate system at the discrete location $q_{n}$. For the given permission precision $\sigma$, In the time period $[(g-1) T, g T]$, over approximate flow pipeline volume $V L_{n g}^{\prime}$ of real system and over approximate flow pipeline volume $V L_{n g}$ of approximate system are calculated by three dimensional over approximate flow pipeline volume algorithm.

(3) $V L_{n}^{\prime}=V L_{n 1}^{\prime}+V L_{n 2}^{\prime}+\ldots V L_{n h_{n}}^{\prime}$ ,$V L_{n}=V L_{n 1}+V L_{n 2}+\ldots V L_{n h_{n}}$. According to the formula $V_{n+1}^{\prime}=V_{n}^{\prime}+V L_{n}^{\prime}$ and $V_{n+1}=V_{n}+V L_{n}$, we can get the new volume sum $V_{n+1}^{\prime}$ and $V_{n+1}$.

(4) For the given permission precision $\varepsilon$, if $\left|\frac{V_{n+1}^{\prime}}{V_{n+1}}-1\right| \geq \varepsilon$, then the real trace and approximate trace are not approximate, trace approximate computation is stop. If $\left|\frac{V_{n+1}^{\prime}}{V_{n+1}}-1\right|<\varepsilon$, then the real trace and approximate trace are approximate. At this moment, if $n=k$, then trace approximate computation is stop. Otherwise it returns step (2) to calculate.

Theorem 1 .If all traces of two three dimensional t-model nonlinear algebraic Hybrid Systems are approximate, then two three dimensional t-model nonlinear algebraic Hybrid Systems are approximate.

The approximate of three dimensional t-model nonlinear algebraic Hybrid Systems not only can optimize three dimensional t-model nonlinear algebraic programs and be able to optimize three dimensional t-model nonlinear algebraic programs and improve computation speed of three dimensional t-model nonlinear algebraic Hybrid System.

\section{Approximate Completed Trace equivalence of Three Dimensional t-Model Nonlinear Algebraic Hybrid Systems}

A completed trace of a three dimensional t-model nonlinear algebraic Hybrid System is a sequence of actions such that $S_{1} \rightarrow^{l_{1}} S_{2} \rightarrow^{l_{2}} \ldots \rightarrow^{l_{n-1}} S_{n}(n>1)$ and $s_{n}$ 
cannot execute any action. Two three dimensional t-model nonlinear algebraic Hybrid Systems are completed trace equivalence if they are trace equivalence and have the same completed traces.

Let $H_{1}$ be a real three dimensional t-model nonlinear algebraic Hybrid System, it obtains the approximate system $H_{2}$ of $H_{1}$ through above mentioned method. Next, it obtains the completed trace equivalence system $\mathrm{H}_{3}$ of $H_{2}$ through the completed trace equivalence theory. $H_{1}$ and $H_{3}$ are approximate completed trace equivalence.

Through above discussion, the approximate completed trace equivalence of three dimensional t-model nonlinear algebraic Hybrid Systems not only can optimize three dimensional t-model nonlinear algebraic programs and be able to optimize three dimensional t-model nonlinear algebraic Hybrid Systems by reducing the states of three dimensional t-model nonlinear algebraic Hybrid Systems.

\section{Experiments}

Let $x_{1}, x_{2}, x_{3}$ respective be horizontal position, relative vertical position, vertical speed of table tennis. $X=\left(x_{1}, x_{2}, x_{3}\right)^{T}$ expresses variables value of table tennis motion system.

$r s=<q, X, f$, Inv $>$, ex $=<e, r, l a b>$. The real table tennis motion system is shown in Figure 5.1.

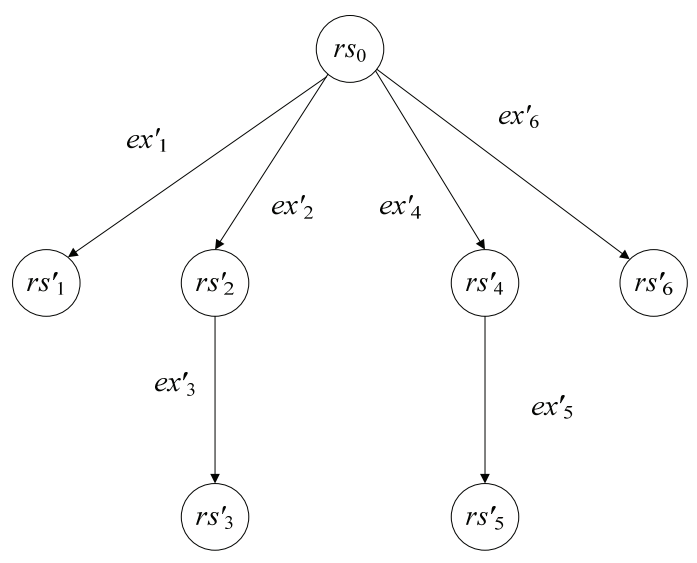

Figure 5.1. real table tennis motion system

The initial state value set of system is $Y_{0}=\left\{\left(0 \leq x_{1} \leq\right.\right.$ $\left.0.1) \wedge\left(23 \leq x_{2} \leq 24\right) \wedge\left(0 \leq x_{3} \leq 0.1\right)\right\}, \operatorname{lab}_{i}(i=1,2,4,6)$ is $X^{\prime}=X, l a b_{j}(j=3,5)$ is $X^{\prime}=\left(\begin{array}{ccc}1 & & \\ & 1 & \\ & & -0.8\end{array}\right) X$

real three dimensional $\mathrm{t}$-model nonlinear algebraic programs $f_{1}^{\prime}$ and $f_{2}^{\prime}$ are $X^{\prime}=X+\left(\begin{array}{c}3.01 t \\ -3.01 t \\ 6.02 t\end{array}\right)$, $f_{3}^{\prime}$ is $X^{\prime}=X+\left(\begin{array}{c}3.01 t \\ 14.448 t-3.01 t^{2} \\ 6.02 t\end{array}\right)$ $f_{4}^{\prime}$ and $f_{6}^{\prime}$ are $X^{\prime}=X+\left(\begin{array}{c}2.99 t \\ -2.99 t^{2} \\ 5.98 t\end{array}\right)$

$f_{5}^{\prime} \quad$ is $\quad X^{\prime}=X+\left(\begin{array}{c}2.99 t \\ 14.352-2.99 t^{2} \\ 5.98 t\end{array}\right)$, invariant set $\operatorname{Inv}_{i}(i=1,2,4,6) \quad$ is $\left\{\left(0 \leq x_{1} \leq 13\right) \wedge\left(0 \leq x_{2} \leq 24\right) \wedge\left(0 \leq x_{3} \leq 19\right)\right\}$, invariant set $\operatorname{Inv}_{j}(j=3,5)$ is $\operatorname{Inv}_{i}(i=1,2,4,6)$ is $\left\{\left(0 \leq x_{1} \leq 13\right) \wedge\left(0 \leq x_{2} \leq 24\right) \wedge\left(-14.4 \leq x_{3} \leq 0\right)\right\}$, It is setting every passing time $T=1$ to calculate flow pipeline in all invariant sets. Compared to the real three dimensional t-model nonlinear algebraic Hybrid System, approximate system has the same conditions except for the different three dimensional $t$-model nonlinear algebraic programs. The approximate three dimensional t-model nonlinear algebraic program

$f_{i}(i=1,2,4,6)$ is $X^{\prime}=X+\left(\begin{array}{c}3 t \\ -3 t^{2} \\ 6 t\end{array}\right)$,

$f_{j}(j=3,5)$ is $X^{\prime}=X+\left(\begin{array}{c}3 t \\ 14.4-3 t^{2} \\ 6 t\end{array}\right)$, Let the given permission precision $\sigma=0.05$ and $\varepsilon=0.03$, volume value is accurate to six significant digits. Through three dimensional t-model nonlinear algebraic Hybrid System trace approximate algorithm, we get $V L_{1}^{\prime}=V L_{2}^{\prime}=0.248251, V L_{4}^{\prime}=V L_{6}^{\prime}=0.249963$, $V L_{3}^{\prime}=0.370508, \quad V L_{5}^{\prime}=0.373482$, $V L_{1}=V L_{2}=V L_{4}=V L_{6}=0.249072$, $V L_{3}=V L_{5}=0.372416, \quad\left|\frac{V L_{i}^{\prime}-V L_{i}}{V L_{i}}\right|<\varepsilon$, where $i=1,2,3,4,5,6$. The real three dimensional t-model nonlinear algebraic Hybrid System and approximate three dimensional t-model nonlinear algebraic Hybrid System are approximate. The approximate system can replace the real system to conduct the scientific research.

According to the approximate completed trace equivalence theory, we can get the approximate completed trace equivalence system of real table tennis motion system which is shown in Figure 5.2.

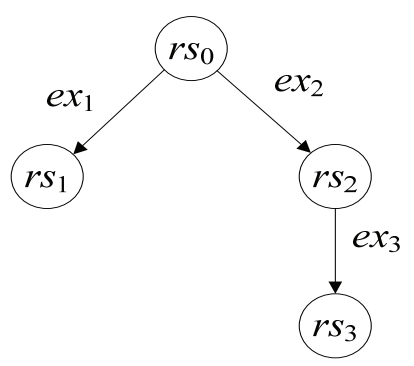

Figure 5.2. approximate completed trace equivalence system of real table tennis motion system

From above computation and analysis, we know that the approximate completed trace equivalence of three dimensional t-model nonlinear algebraic Hybrid Systems can optimize three dimensional t-model nonlinear 
algebraic program as well as reduce the number of system states.

\section{Conclusion}

In this paper, the approximate completed trace equivalence of three dimensional t-model nonlinear algebraic Hybrid Systems is proposed. Under certain conditions, it can reduce coefficient bit of three dimensional t-model nonlinear algebraic programs and eliminate states of three dimensional $\mathrm{t}$-model nonlinear algebraic Hybrid Systems. In the future work, we will study the approximate completed trace equivalence of high dimensional t-model nonlinear algebraic Hybrid Systems.

\section{Acknowledgement}

This work is partially supported by Grants HCIC201101 of Guangxi Key Laboratory of Hybrid Computational and IC Design Analysis Open Fund, the Fundamental Research Funds for the Central Universities of Lanzhou University under Grant No. 860772, the National Natural Science Foundation of China under Grant No. 60973147, the Doctoral Fund of Ministry of Education of China under Grant No. 20090009110006, the Natural Science Foundation of Guangxi under Grant No. 2011 GXNSFA018154, the Science and Technology Foundation of Guangxi under Grant No. 10169-1, and Guangxi Scientific Research Project No.201012MS274.

The authors are grateful to the anonymous referee for a careful checking of the details and for helpful comments that improved this paper.

\section{References}

[1] Fabien Lauer, Gérard Bloch, René Vidal, A continuous optimization framework for hybrid system identification, Automatica, 47, 608-613 (2011).

[2] Suzuki, Tatsuya , Advanced motion as a hybrid system, Electronics and Communications in Japan, 93, 35-43 (2010).

[3] Carol Mansour, Denis Clodic, Dynamic modeling of the electro-mechanical configuration of the Toyota Hybrid System series/parallel power train, International Journal of Automotive Technology, 13, 143-166 (2012).

[4] Bin Wu, Yongjun Fu, Generating Invariants for Hybrid Systems by Computing Vanishing Ideals of Sample Points, JDCTA, 6, 252-258 (2012).

[5] Hao Yang, Jinzhao Wu, Zhiwei Zhang, Approximate Completed Trace Equivalence of Inhomogeneous Linear Transition SystemsInternational Journal of Advancements in Computing Technology, 4, 58-66 (2012).

[6] Hao Yang, Jinzhao Wu, Yang Liu, Zhiwei Zhang. Approximate Completed Trace Equivalence of Homogeneous Linear Algebraic Hybrid SystemsJournal of Convergence Information Technology, 8, 520-527 (2013).
[7] A. Chutinan, B. H. Krogh, Computational techniques for hybrid system verification, IEEE Transactions on Automatic Control, 48, 64-75 (2003).

[8] A. Chutinan, Hybrid system verification using discrete model approximations, Carnegie Mellon University, (1999).

[9] J. Li, The methods of linear Hybrid System formal verification and its application, Hefei University of Technology, (2009).

[10] B. Zhang, The research on formal verification of Hybrid Systems, Hefei University of Technology, (2007).

[11] B. Mandal, S. Adhikari, K. Choudhary, A. Dey, A. Biswas, A. K. Bandyopadhyay, A .K. Bhattacharjee, D. Mandal, Femtosecond response of quantum discrete breathers in SRR based metamaterials and the role of dielectric permittivity, Quantum Physics Letters, 1, 59-68 (2012).

[12] P. K. De, A. Bhincher, Dynamic programming and multi objective linear programming approaches, Applied mathematics \& Information Sciences, 5, 253-263 (2011).

[13] D. Kumar, M. I. Khan, Relations for Generalized Order Statistics from Doubly Truncated Generalized Exponential Distribution and its Characterization, Mathematical Sciences Letters, 2, 9-18 (2013).

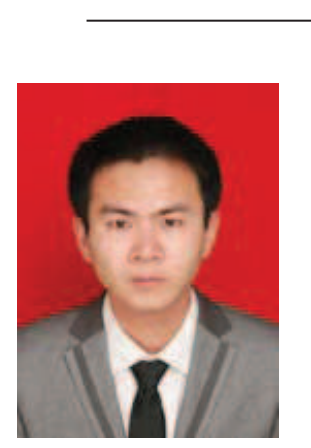

Hao Yang is a doctorial student in Chengdu Institute of Computer Application, Chinese Academy of Sciences, China. He has published more than two articles in reputed international journal of software engineering and computer sciences. His research interests are in the areas of formal verification and network security

Jinzhao Wu is currently a professor in Guangxi University for Nationalities and Beijing Jiaotong University. His research interests lie in formal verification and software engineering.

Zhiwei Zhang is a doctorial student in Chengdu Institute of Computer Application, Chinese Academy of Sciences, China. His research interests are in the areas of formal verification and network security.

Yang Liu is a doctorial student in Chengdu Institute of Computer Application, Chinese Academy of Sciences, China. Her research interests are in the areas of formal verification and network security. 